\title{
AUCH GEHÖRLOSE LERNER KÖNNEN FREMDSPRACHEN LERNEN
}

\author{
BARBARA SKOWRONEK
}

\begin{abstract}
How do the deaf people learn to communicate? Can the deaf people learn the phonic language (sound language, germ. lautsprachlich kommunizieren); can they learn foreign languages? What conditions should be met for this? What support should they expect from the family, from teachers, from hearing people? The answer to this will be a description of Sarah Neef's learning the sound language.
\end{abstract}

Key words: communication, foreign languages learning for deaf people

\section{Der Mensch - Kommunikation - Sprache - Wissen}

Kommunikation ist Ausdruck menschlicher Relationen zwischen den Sendern und den Empfängern, es ist das menschliche Bedürfnis, andere zu kontaktieren, zur primären und sekundären Orientierung in der Umwelt. Dazu ist der Mensch mit Wissen ausgerüstet, einer angeborenen Grundeigenschaft, der Kommunikationsfähigkeit, um seine Lebensfunktionen zu erhalten: sich der Umwelt anzupassen (als Erkenntnispotential) (vgl. Grucza, 2012). Wissen existiert als angeborenes Erkenntnispotential eines Menschen, genetisch vererbbar, um sich der Umwelt (Umgebung, Verkehrsgemeinschaft) anzupassen; dadurch ist der Mensch kommunikativ erkenntnisfähig. Wissen existiert (ebenso) als angeborenes Entwicklungspotential des Menschen, genetisch kaum vererbbar, um die Umwelt zu beeinflussen; es ist des Menschen (Lern)Fähigkeit, sich (und seine Umwelt) zu entwickeln, also zu lernen; es erlaubt dem Menschen kommunikativ lernfähig, entwicklungsfähig zu sein; dieses Wissen muss jeder selbst erarbeiten, rekonstruieren, generieren, um zu erlernen. Wissen ermöglicht dem Menschen, sich sprachlich und nichtsprachlich zu verständigen, mit Hilfe von Diskurs, Texten, als den geordneten kulturdeterminierten 
sprachlichen Mitteln (als lexikalische, morphosyntaktische, semantische Ausdruksweisen, Pläne, Schemata, Normen, Konventionen, kommunikative Regeln, feste Ausdrucksmittel und Verhaltensweisen, Olpińska, 2009), je nach den Formationsregeln (phonemisches und grammatisches Wissen) und Funktionsregeln (semantisches und pragmatisches Wissen) (Grucza, 1993: 150).

Sprache ist ein kulturdeterminiertes Kommunikationsmittel; es dient nicht nur zur Vermittlung von Information, auch zum Ausdruck von Emotionen und psychischen Zuständen. Wirkliche menschliche Sprachen existieren als kulturdeterminiertes Wissen des konkreten Menschen (kognitiv) sowie im Austausch von zwischenmenschlichen Kontakten (kommunikativ) (Bonacchi, 2011). Sprache lässt sich (idiolektal) als eine innere mit dem konkreten Menschen integrierte kulturdeterminierte Fähigkeit auffassen, die dem konkreten Menschen ermöglicht, intersubjektiv mit anderen Menschen (polylektal) zu kommunizieren. Daneben lässt sich Sprache als propotypische Modelle/Abbildungen auffassen (Grucza, 2012).

Sprache ist mit Kultur verbunden - beide existieren als dynamisches Gefüge interagierender menschlicher Fähigkeiten, im Gehirn des jeweiligen Menschen lokalisiert und kognitiv verarbeitet, für den intersubjektiven Gebrauch (mit anderen) kommunikativ da. Der Mensch ist also sowohl ein individuell wie auch gesellschaftlich sprachlich-kulturell (und nichtsprachlich) handelndes Kommunikations-Subjekt. Dadurch dass Sprache kulturdeterminiert ist, macht Sprache uns Menschen, gesellschaftliche Wesen (homo socius) zu Kulturwesen (homo cultus). Folglich muss auch das Sprachen-Lernen kulturdeterminiert verlaufen. Sprache ermöglicht die ganze Bandbreite des menschlichen Denkens und Handelns auszudrücken und wahrzunehmen. Jeder rationale und logische Denkvorgang als Wissensvermittlung vollzieht sich unter Mitwirkung von Sprache. Wissen lässt sich nur mit Hilfe von Sprache festhalten. Die Vielfältigkeit der Sprachen und Dialekte drückt eine immense Palette unterschiedlicher Weltansichten, Literaturen und Lebensweisheiten aus. Sprache ist also auch Ausdruck der Identität, der gesellschaftlichen Zugehörigkeit eines jeden Menschen, das Mensch-Sein, seine „Visitenkarte“. Sprache signalisiert, wer wir sind und wo wir hingehören, es ist eine Möglichkeit, sich (oft auch unbewusst) auszuweisen, über sprachliche Varietät, regionale Herkunft, sozialen Hintergrund, Bildungsniveau, Alter usw., über unsere Zugehörigkeit Auskunft zu geben; manchmal bindet sie mehr ein, als dass sie informiert (bei emotionellen Ereignissen der Zusammenfügung, sehr deutlich erkennbar in Telefongesprächen).

Der Mensch ist ein sprach-, kultur- und wissensgenerierendes Wesen (Grucza 1993: 15), ausgerüstet mit einem Erkenntnispotential und Entwicklungspotential. Das Grundbedürfnis des Menschen ist, sich in der Umwelt (Verkehrsgemeinschaft) zu orientieren, also (primär) sich der Gemeinschaft anzupassen und (sekundär) möglichst die Gemeinschaft zu beeinflussen. Dazu müssen Kontakte, Relationen zu anderen geknüpft werden, das bedeutet, sprachlich und nichtsprachlich zu kommunizieren (homo communicans). 


\section{Das Lernen}

Lernen lässt sich (nach Grucza, 2012; Olpińska, 2009) als autonomes Wahrnehmen von Wissen seitens des Lerners auffassen: kognitives Generieren, Rekonstruieren des vom Lerner konstruktiv Wahrgenommenen. Die Rolle des Lehrers ist nur, Wissen initiierend zu präsentieren, und Bedingungen für konstruktive Selbständigkeit des Lerners zu schaffen. Das eigentliche Lernen muss der Lerner selbst vollbringen (dazu ist der Mensch mit dem Erkenntnispotential und Entwicklungspotential ausgestattet). Kommunikationsfähigkeit erlaubt dem Menschen, sich in der Umwelt zu orientieren: sich anzupassen (im Rahmen des Erkenntnispotentials) und die Umwelt zu beeinflussen (im Rahmen des Entwicklungspotentials). Kommunikationsfähigkeit umfasst auch Sprachlernfähigkeit. Das sprachliche (und nichtsprachliche) Miteinander ist kommunikativer Ausdruck zwischenmenschlicher Relationen und der Gemeinschaftszugehörigkeit. Jeder Mensch braucht die Zugehörigkeit zu einer Gruppe, einer Gemeinschaft, ohne die er minderwertig vereinsamen kann. Jedem Menschen ist Kommunikationsfähigkeit angeboren, um sich in der Verkehrsgemeinschaft zu orientieren: um mit anderen Kommunikationsmitgliedern zu handeln, sich der Gemeinschaft anzupassen und die Gemeinschaft zu beeinflussen. Dazu braucht der Mensch auch sprachliche und nichtsprachliche Kommunikationsmittel, die erlernt werden müssen. Sprachfähigkeit lässt sich als das menschliche Bedürfnis erklären, mit Hilfe von Sprache zu kommunizieren. Dazu muss Sprache erlernt werden.

Der Mensch lernt, indem er die Wirklichkeit mit Sinnen (sensorisch) wahrnimmt: die wahrgenommenen Eindrücke werden ins Gehirn geleitet, wo sie kognitiv verarbeitet, zur Lern- und Lebenserfahrung des Menschen beitragen. Deswegen ist das gesunde Funktionieren einzelner Sinnesmodalitäten wichtig (Sehen, Hören, Riechen, Tasten, Schmecken; Intuition). Es wird gesehen, gehört, gerochen, gefühlt, getastet, geschmeckt. Augen, Ohren usw. nehmen Eindrücke aus der Umgebung auf, verarbeiten sie und leiten ins Gehirn weiter, wo sie kognitiv verarbeitet werden, um im Gedächtnis effektiv zu landen. Daher ist das korrekte Funktionieren der einzelnen Sinne für die kognitive Wahrnehmung der Wirklichkeit von größter Bedeutung, folglich für gegenseitige zwischenmenschliche Kontakte, auch für das Lernen.

Der Mensch kommt zur Welt mit zwei Ohren (als Hörorgan), zwei Augen (als Sehorgan) und einem Mund (als Sprechorgan); das ermöglicht ihm, die Welt hörend, sehend und sprechend wahrzunehmen, um zweimal so viel zu hören und zu sehen als zu sprechen. In der Gesellschaft wird erwartet, (laut)sprachlich zu kommunizieren, um in eine bestimmte Verkehrsgemeinschaft aufgenommen zu werden. Wer nicht sprechen kann, wird oft für nicht vollwertig, kontaktunfähig genommen. Sensorische Dysfunktionen wirken sich negativ auf die Kommunikationsfähigkeit und Kommunikationsmöglichkeit des Menschen aus.

Bei Störung eines Sinnes (oder mehrerer Sinne) ist der Zugang zur Wahrnehmung der Wirklichkeit erheblich erschwert (oder gestört). So kann bspw. die Fehl- 
funktion des Hörorgans zur Gehörlosigkeit führen, was den betroffenen Gehörlosen sogar aus der Verkehrsgemeinschaft ausschließen lässt. Das unkorrekte Funktionieren eines Sinnes kann durch das vermehrte Funktionieren eines anderen Sinnes ausgleichend kompensiert werden, bspw. wer nicht sehen kann, ist empfindlicher für Geräusche, für Tastsinn. Wer nicht hören kann, ist in seiner Aufnahme der gesamten Geräuschkulisse wesentlich beeinträchtigt, was sich negativ auf seine lautsprachliche Ausdrucksweise auswirkt: trotzdem seine Sprechorgane gesund sind und korrekt funktionieren könnten, kann er nicht sprechen.

Das Kind mit Hörschaden ist eingeschränkt in seiner ganzen Erfahrungswelt durch die Verringerung bzw. das Fehlen des akustischen Reizangebots, was gleichzeitig das Fehlen des Anstoßes für die Sprachentwicklung bedeutet. Dadurch droht ihm Abgeschnittensein, Separation von seiner hörenden Umgebung. Um dem entgegenzuwirken, muss versucht werden, gehörlosen Kindern akustische Reize (Laute) zukommen zu lassen. Umso mehr, weil nur ihr Hörorgan defekt ist, die Sprechorgane sind nicht defekt. Das bedeutet, der kommunikative Lernprozess des gehörlosen Kindes sollte so gesteuert werden, damit das Sprechen genutzt werden kann. $\mathrm{Zu}$ erarbeiten werden sollten solche Bedingungen, die das Sprechen Gehörloser aktivieren lässt. Gehörlose können sprechen lernen. Die Frage ist nur: Wie? Das bedeutet auch, dass der Ausdruck taub-stumm nicht berechtigt ist.

\section{Gehörlosigkeit}

Gehörlosigkeit ist eine Behinderung, die schon ein Kleinkind in seiner gesamten geistigen, kognitiven und emotionalen Entwicklung schwer beeinträchtigt und enorme Kommunikationsschwierigkeiten bereitet. Immer noch herrscht die verbreitete Meinung, (die angeborene oder frühzeitig erworbene vollständige) Taubheit führe zu Stummheit, also zur Kommunikationsunfähigkeit (folglich: Dummheit). Dieses beklemmende Vorurteil ist noch weit verbreitet; es ist unfair, ungerecht und niederschmetternd, vor allem, weil es nicht stimmt! Diesen ungerechten Meinungen sollte man entgegentreten: Das Leben zeigt, dass Menschen mit (unterschiedlichsten) sensorischen Dysfunktionen genetisch mit anderen außerordentlichen Befähigungen ausgestattet, ausgeglichen werden: bspw. Blinde oder schwer Sehbehinderte sind mathematisch talentiert (im logischen Denken), ausgerüstet mit sehr hohem I-Quotienten! Gehörlose sind mit mehr Empfindlichkeit für Berührungen und Vibrationssensibilität ausgestattet, dazu kommt ihre enorme Beobachtungsfähigkeit und breitperspektivisches Sehen, als Ausgleich und Ergänzung der fehlenden Seh- oder Hör-Wahrnehmungsmöglichkeiten. Diese Kompensationen als „Zusatzgaben“ können ihnen reichlichen Zugang auch zur Berufswahl geben.

Gehörlosigkeit ist Dysfunktion des Hörorgans bis zur Taubheit. Gehörlosigkeit bedeutet Nicht-(oder Schwer-)Hören-Können, folglich die Unfähigkeit, Laute nach- 
zuvollziehen und auszusprechen. Gehörlosigkeit verursacht auditive Probleme: Wer nicht Laute produzieren kann, kann die mediale Mündlichkeit nicht realisieren, ohne die es unmöglich ist, an Gesprächen teilzunehmen (konzeptionelle Mündlichkeit, Diskursfähigkeit).

Für Hörende vollzieht sich der Hörprozess ganz natürlich, nebenbei, so instinktiv wie das Atmen: Geräusche der Umwelt werden (dank dem gut funktionierenden Hörorgan) automatisch wahrgenommen, das Kind hört zu, versucht, sich durch Nachahmung und durch ständige Selbstkontrolle (und Bewertung der Hörenden und seines Sprechens), mit seinen produzierten Lauten, den Lauten seiner Umgebung anzupassen.

Gehörlosigkeit gilt als die stärkste soziale Behinderung (angeblich stärker als Seh-Defizite), die die Wahrnehmung und Orientierung in der Umgebung beeinträchtigt. Sie führt zur kommunikativen Isolation und Vereinsamung Gehörloser, die erst im Kontakt mit anderen Gehörlosen entweder mit Gebärdensprache oder mit Lautsprache - Mut fassen, um aktiv zu leben zu beginnen. Gehörlosigkeit kann Sprachlosigkeit und Kommunikationsschwierigkeiten, gar Kommunikationslosigkeit bedeuten; Wer nicht sprechen kann, sich nicht mitteilen kann, wird häufig für sprachlos, für stumm=unverstanden=dumm gehalten. Darunter leiden v.a. Gehörlose, die unfair betrachtet werden. Umso mehr, weil in der Welt der Hörenden lautliches Sprechen verlangt wird.

\section{Gehörlosenpädagogik, surdopedagogika}

Mit Gehörlosigkeit beschäftigt man sich in Deutschland im Rahmen der Gehörlosenpädagogik (Hörgeschädigtenpädagogik) als dem Teilgebiet der Sonderpädagogik und der Audiologie. In Polen heißt die Disziplin, innerhalb derer man sich mit Gehörlosigkeit beschäftigt, surdopedagogika (lat. surdus bedeutet gehörlos). Das Ziel der Gehörlosenpädagogik ist die fachspezifische Erziehung und Bildung von Kindern und Jugendlichen mit Gehörlosigkeit bzw. Schwerhörigkeit von der Früherziehung bis zur beruflichen Eingliederung sowie Erwachsenenbildung und Familienbetreuung. Ziel dieser Disziplin ist theoretische und praktische Beschäftigung mit Gehörlosigkeit (v.a. Diagnostik und Profilaxe), um den Patienten integrativen Zugang zur Verkehrsgemeinschaft der Hörenden zu gewährleisten, Unterstützung in Bildung und Erziehung sowie Hilfe den Familien der Gehörlosen ausgleichend zu bieten. Es werden zahlreiche Programme zur Frühförderung in Bildung und Berufswahl für Gehörlose entwickelt, die den Betroffenen (und ihren Familien) verhelfen, am Leben aktiv teilzunehmen. Die heutige Medizin bietet den Gehörlosen Hilfen u.a. Hörgeräte und Hörimplantate, die den Patienten zur audialer Wahrnehmung der Wirklichkeit mindestens annähernd verhelfen sollen. Früher war die Gebärdensprache die (ziemlich einzige) kommunikative Kontaktmöglichkeit; heute wird die laut- 
sprachliche Erziehung angestrebt, um Gehörlosen bessere Integration in der (hörenden) Gesellschaft sowie berufliche Eingliederung zu ermöglichen. Es wird mit Hilfe der Gebärdensprache oder lautsprachlich kommuniziert (oder beides).

\section{Polnische Gebärdensprache, Gebärdensprache-Linguistik}

Mit der Polnischen Gebärdensprache (Polski Język Migowy, PJM) beschäftigt man sich wissenschaftlich im Rahmen der Gebärdensprache-Linguistik an der Universität Warszawa (vgl. Świdziński, Rutkowski, Mikulska, Kołodziejska), an der Universität Gdańsk (Żukowska). Rutkowski (2017) ist entschiedener Befürworter der Gebärdensprache. Dafür bereitet er Lehrmaterialien mit Filmen für die einzelnen Unterrichtsfächer für gehörlose Lerner mit der Gebärdensprache vor. Er weist darauf hin, dass viele Eltern die Gebärdensprache als stigmatisierend sehen (weil sie Gehörlosigkeit sichtbar macht), und sich weigern, die Gebärdensprache ihren Kindern zu vermitteln.

Mikulska erarbeitete (an der Universität Warszawa) Baby-Gebärden (pol. bobomigi oder gadajace paluszki also dt. plappernde Fingerchen/ Händchen"), die mit Erfolg nicht nur für gehörlose, sondern auch für hörende Säuglinge als kommunikatives Training von der Autorin eingesetzt werden. Dieses sehr interessante Gebärdensprache-Training verhilft besonders den gehörlosen Säuglingen bei ihrer allgemeinen gesellschaftlichen, kognitiven und emotionalen Entwicklung, um später zu aktiven, vollwertigen Kommunikationsteilnehmern, ohne Minderwertigkeitskomplexe, heranzuwachsen. Dieses Vorhaben ist voll zu unterstützen: Bekanntlich sind die Gebärden eine natürliche Vor-Etappe der Kommunikation für alle Kleinkinder (und mit Kleinkindern), die mit ganzem Körper kommunizieren und sich so dem Geräusche-Rhythmus ihrer Umgebung anpassen. Daher ist es nur zu begrüßen, diese natürliche Phase für alle Kleinkinder anzuwenden, um sie für die gesamte Entwicklung des Kindes auszunutzen. Dieses Gebärden-Training ist auch hörenden Säuglingen eine effiziente Vorbereitung und Stütze nicht nur für deren allgemeine gesellschaftliche, kognitive und emotionale Sprachentwicklung, sondern auch auf ihren weiteren lautlichen Spracherwerb, also auf Zweitsprachenerwerb und Fremdsprachenunterricht bestens vorbereitet. Inzwischen gibt es in Polen mehrere engagierte Personen, die mit Baby-Gebärden arbeiten: Kołodziejska (Uniwersystet Warszawski), Żukowska (Uniwersystet Gdański).

\section{Die Rolle des Elternhauses}

Die Rolle des Elternhauses ist, den gehörlosen Kindern kommunikativen Zugang zu Hörenden zu verschaffen. Es ist eine enorme Herausforderung und Verantwortung (eigentlich böses Erwachen). Sie müssen sehr schnell, möglichst in den ersten Mona- 
ten, über die Zukunft ihres Kleinkindes entscheiden, damit es lebenslang vollwertiges, autonomes, kreatives Gesellschaftsmitglied sein kann: über Kommunikationsfähigkeit, Bildung, soziale Entwicklung, Beruf. Die Eltern müssen die Entscheidung für die kommunikative Entwicklung ihres gehörlosen Kindes treffen. Umso mehr, weil viele (die meisten) Eltern und Lehrer von gehörlosen Kindern nicht gehörlos sind: sie können hören, also auch lautlich sprechen; wenn sie sich für die Gebärdensprache für ihr Kind entscheiden, müssen sie die Gebärdensprache auch selbst erst erlernen.

Als Allerwichtigste scheint, dem Kleinkind zu verhelfen, seine Dysfunktion zu akzeptieren, um eine bessere Lebensqualität anzustreben, und die eigene Identität zu bewahren. Gehörlosigkeit ist für den Betroffenen (und dessen Familie meistens) bleibende Tatsache, objektiver Sachverhalt: man ist und bleibt taub. Das heißt aber auch, sich nicht durch das Prisma seiner sensorischen Defizite bedrücken zu lassen, sondern zu erkennen, was man außergewöhnlich von Mutter Natur geschenkt bekommen hat, also mit welchen Fähigkeiten man angeboren ausgestattet ist, um sich prinzipiell des Lebens zu erfreuen. Das bedeutet, dankbar dafür zu sein, was man hat, und nicht undankbar dafür, was man nicht hat! Gehörlosigkeit mag zwar „ein Hemmnis in der Entwicklung darstellen, aber es lässt sich überwinden" (Neef 2009: 42). Sehr wichtig ist Unterstützung und Zuspruch der Nächsten, um erfahren zu können, dass diese Dysfunktion keine Gefängnismauer darstellt, die von der Welt abschneidet, sondern den Zugang zur Wahrnehmung der Welt Hörender nur erschwert. Dazu ist Familie da (als unschätzbares Gut).

Die Eltern eines gehörlosen Kindes müssen sich als Hörende mit Gehörlosigkeit sehr schnell anfreunden, Empathie als die Vorstellung von dem Nicht-Hören-Können entwickeln, damit der Kommunikations-Unterricht für das Kleinkind beginnen kann und damit ihr gehörloses Kind keine Schäden als Folgen von sensorischen Entwicklungsstörungen trägt; all das, um dem Kind den kompensierten Zugang zur aktiven Wahrnehmung der Welt und Orientierung in der Welt zu ermöglichen. Deswegen müssen sich Eltern von gehörlosen Kleinkindern sehr schnell auf jene Sinne konzentrieren, die beim Kind korrekt funktionieren, um diese Sinne hervorhebend, ausgleichend und kompensierend zu gebrauchen, damit die Kinder akzeptiert, anerkannt (und nicht nur geduldet, geschweige denn diskriminiert) werden können. Auch Gehörlose haben das Recht, glücklich zu sein!

Gehörlosigkeit erfordert entsprechende kommunikative Ausgleichsbedingungen für das Kontaktieren mit Hörenden, damit sich Gehörlose als vollwertige Mitglieder der sprachlich-kulturellen Verkehrsgemeinschaft verständigen können: verstehen und verstanden, respektiert und akzeptiert werden. Welchen Weg der allgemeinen Entwicklung das Kind einschlagen soll, um lebenslang selbständig und beruflich abgesichert zu sein: ob als Manualist (dann muss es die Gebärdensprache lernen) oder als Oralist (dann muss es das Lippenlesen und Produzieren von Lauten lernen). Beide Kommunikationsmöglichkeiten, Gebärdensprache und Lautsprache sind die Kommunikationsmöglichkeit für Gehörlose, sie haben Sprache als Kommunikationsmittel zum Ziel, also Texte mit Zeicheninformationen, die aus Form und Inhalt bestehen, zu ver- 
stehen und zu produzieren: Manche Gehörlose können sowohl die Gebärdensprache als auch das Lippenlesen. Manche Manualisten können nur die Gebärdensprache und bleiben Manualisten; manche Oralisten können nur die Lautsprache und bleiben Oralisten. Das Hauptziel muss sein, gehörlosen Kindern sehr bald kommunikativen $\mathrm{Zu}-$ gang zu hörenden Menschen ihrer näheren und/oder weiteren Umgebung zu ermöglichen, lebenslang effiziente, leistungsfähige Werkzeuge zum Kommunizieren zu geben, um die Welt wahrzunehmen, zu erkennen, um sich aktiv und selbständig zu orientieren, um all das lernen zu können, was auch hörende Kinder können.

Übrigens: hörende Kinder gehörloser Eltern, die nur durch die Gebärdensprache kommunizieren, lernen die Gebärdensprache ihrer Eltern als Erstsprache $=$ Muttersprache. Erst nach und nach (im Kindergarten) lernen sie dann die Lautsprache, sozusagen als erste Fremdsprache, weil sie hören können, also nicht taub sind. Die Gebärdensprache nutzen sie später oft aus, um zwischen gehörlosen Manualisten und Hörenden zu dolmetschen, zu vermitteln, eventuell auch als Lehrer für Manualisten zu sein.

\section{Sarah, ein Beispiel für FSU an Gehörlose}

(Wie) Ist es möglich, gehörlos lautsprachlich zu kommunizieren? (Wie) Ist es möglich, gehörlos Fremdsprachen zu lernen? Am Beispiel von Sarah Neef, einer gehörlosen Lernerin, soll präsentiert werden, dass es möglich ist, lautsprachlich zu kommunizieren.

Sarah Neef, 37, ist als eine lautsprachlich kommunizierende Gehörlose (Oralistin) ein positives Beispiel dafür, ihr Leben bestens zu leben: sie ließ sich von ihrer krankhaften Dysfunktion nicht unterdrücken! Dank der lautsprachlichen Erziehung ist Sarah eine sprechende Gehörlose, die Folgendes erreicht hat: Nach einer lautsprachlich geprägten Erziehung im Elternhaus bestand sie zunächst ihr Abitur und schloss ihr Psychologiestudium ab, beides mit Bestnoten. Trotz ihrer Gehörlosigkeit beherrscht sie nicht die Gebärdensprache, dafür aber außer Deutsch als Muttersprache auch einige Fremdsprachen (Englisch, Latein, Französisch und Russisch), die sie in der Schule gelernt hat. Ihre Leidenschaft ist das Tanzen, das sie letztens als Lehrerin und Choreografin betreibt. Sie ist als promovierte Psychologin mit Erfolg berufstätig. Auch den Führerschein hat sie gemacht und fährt Auto. Telefon besitzt sie nicht, Handy nur, um SMS schreiben und empfangen zu können.

Aus dem Buch, das Sarah 2009 veröffentlicht hat, erfahren wir, Sarah hat infolge von Entbindungs-Schwierigkeiten ihr Hörvermögen verloren. Zwar kam sie mit dem ersten Schrei, lebhaftem Blick der wachsamen Augen auf die Welt, doch ihre Eltern merkten, dass Sarah jedes Mal weinte, wenn sie ihre Mutter nicht sah. Der Kinderarzt hielt die Mutter für überbesorgt. Erst mit elf Monaten bekamen ihre Eltern bestätigt: Sarah kann nicht hören! Sarahs Hörverlust betrug 110 bis 120 Dezibel (Düsenflieger, 
dieses Schallereignis treibt die Hörenden an die Schmerzgrenze). Ihre Eltern wählten für Sarah die lautsprachliche Erziehung. Das aus zwei Gründen: Ihre Eltern konnten die Gebärdensprache nicht und durch die Lautsprache wollten sie ihrer Tochter kommunikativen Zugang zur Mehrheit der Menschen, also zu lautsprachlich kommunizierenden Hörenden ermöglichen. Auch wollten sie Sarah zur Selbständigkeit erziehen, damit sie lebenslang autonome Entscheidungen selbst treffen kann. Dafür ist sie ihren Eltern äußerst dankbar. Sarah war von Anfang an Meister der (intuitiven) Kompensation, womit sie ihre Umgebung täuschte: im frühesten Baby-Alter, sich der Umgebung anzupassen und zu reagieren; sie ersetzte die Wahrnehmung der Geräusche durch das Beobachten mit Augen, mit denen sie munter und forschend in die Welt blickte: kompensierend zu erfassen, was um sie geschah. Und reagierte sehr schnell (Karpińska Szaj 2013), was selbst den Kinderarzt verirrte, der Sarahs Mutter belächelte, als sie an der Hörfähigkeit ihres Kindes zweifelte.

Obwohl Sarahs Weg zur kommunikativen (lautsprachlichen) Selbständigkeit steil und überhaupt nicht einfach war, bereut sie nichts (Was mich nicht umwirft, das macht mich stark!). Wahrscheinlich half ihr dabei, sehr bald gelernt zu haben, Dinge, die man nicht beeinflussen kann, anzunehmen und zu akzeptieren, anstatt daran zu verzweifeln. Es ist bewundernswert, dass und wie es ihr gelungen ist, als Gehörlose die Welt der Hörenden zu erkämpfen! Sie ist sich ihrer Behinderung, gehörlos (taub) zu sein, bewusst und akzeptiert es! Gehörlosigkeit ist ihr Markenzeichen, ihr Charakteristikum, sie hat gelernt, damit kompensierend, ausgleichend umzugehen. Das ermöglichte ihr eine Lebensbejahung zu entwickeln, so dass sie mit voller Überzeugung bestätigt, nicht nur eine glückliche Kindheit gehabt zu haben, sondern ihr Leben ziemlich voll in Griff zu haben, sehr gut organisiert, und diszipliniert zu leben.

Sarah liest von Lippen ab. Sie nimmt mit Hilfe von Lippen-Lesen ihr audiales Unvermögen, die Welt der Laute und Geräusche wahr, sie rekompensiert das NichtHören-Können mit Ablesen von Lippen und von der gesamten Körpersprache ihrer Kommunikationspartner, um zu erahnen und zu verstehen, was Hörende meinen; es ermöglicht ihr den Zugang zu Hörenden, die Verbindung zu Menschen, die zwischenmenschliche Kommunikation, gesellschaftliche Relationen einzugehen, die Menschen zu kontaktieren und zu verstehen, möglichst auch verstanden und akzeptiert zu werden. Aber eine besonders große Stütze waren (und sind es weiterhin) ihre Augen: mit Augen entwickelte sie das breitperspektivische Sehen, auch um die Körpersprache ihrer Kommunikationspartner zu beobachten, und um den Klang und die Lautstärke der von ihr produzierten Laute ihrer Umgebung anzupassen. Sarah hat gelernt, mit Augen ihre Sprechweise intuitiv situationsgemäß dem Redefluss ihrer Umgebung anzupassen: an den Reaktionen ihrer Gesprächspartner erkennt sie (bis heute noch), ob sie leiser oder lauter sprechen soll. Augen sind für Sarah eine stellvertretende und ergänzende Kompensation für ihr fehlendes Hören. (auch Karpińska-Szaj 2013, Domagała-Zyśk und Karpińska-Szaj 2011).

Sarah trägt seit ihrer Kindheit ein Hörgerät, zu dem sie lange Zeit eine Hassliebe entwickelt hatte. Dieser „Knopf“ brachte Sarah eine leise Ahnung vom Hören, und 
er half ihr zur Orientierung in ihrer Umgebung. Teilweise verhelfen Hörgeräte auch, die Sensibilität für Vibrationen zu entwickeln, zu unterstützen, zu aktivieren (aber nur teilweise; denn zu laut eingestellt, verursachen sie Schmerzen im Körper des Gehörlosen).

\section{Die Rolle ihrer Eltern}

Ihre Eltern mussten zuerst die entscheidenden Fragen beantworten : Wie soll Sarah die Welt wahrnehmen, erkennen, erlernen? Wie soll sie in der Umwelt funktionieren? Wie soll sie mit ihrer Umgebung kommunizieren? Wie soll man einem Kind die Welt, die voller Geräusche zur Orientierung dienen, die aber nicht in das Ohr eines gehörlosen Kindes eindringen, erklären, beibringen, erklären? Wie wachsen gehörlose Kinder auf? Wie lernen sie? Wie können sie kommunizieren, um akzeptiert zu werden? Wie können gehörlose Kinder zur Selbständigkeit erzogen werden? Was ist die beste Erziehung für das gehörlose Kind? Zu Hause? Im Internat (ohne die Liebe des Elternhauses)? Würde sich Sarah jemals in der Gesellschaft zurechtfinden können oder drohte ihr gänzliche Isolation, das Nicht-Akzeptiert-Sein? Würde sie verstehen und verstanden werden; würde sie akzeptiert und respektiert werden? Soll man Sarah vor allem Bösen der Welt beschützen oder sie darauf vorbereiten, dass es nicht leicht und einfach sein wird? Wie viel Verantwortung lastet auf ihnen, den Eltern?

Diese Fragen müssen sich alle Eltern gehörloser Kinder stellen und beantworten, um der Herausforderung gewachsen zu sein: ihr Kind zur Selbständigkeit zu erziehen, um der Abhängigkeit von anderen möglichst vorzubeugen. Sarahs Eltern wollten ihr die Freiheit im Leben schenken, ihr die Selbstbestimmung, Selbstverwirklichung (Autonomie) überhaupt erst möglich machen. Es musste ein straffer Plan entwickelt werden, auch um Seelentröster und bester Freund für die schweren Momente der Verzweiflung, die kommen würden, zu sein: immer wieder mit schwierigen Situationen fertigzuwerden. Sie brachten ihr sowohl die Grundtugenden bei, wie Liebe, Ehrlichkeit, Herzenswärme, Recht von Unrecht unterscheiden, aber auch Disziplin und Lernbereitschaft. Ebenso gaben sie ihr Mut, Selbstvertrauen und Rückhalt! Sie wollten Sarah eine stilvolle Sprache vermitteln, deshalb achtete (besonders) ihre Mutter auf eine richtige Wortwahl, um Sarah viele Ausdrucksmöglichkeiten zu vermitteln, umfangreicheren Wortschatz zu präsentieren, damit sich Sarah wirkungsvoll ausdrücken kann.

\section{Verlauf ihres Lernens}

Mit elf Monaten begann für Sarah der aktive Sprachunterricht: eigentlich zu spät, landeten sie in der Schweiz bei Susann Schmid-Giovannini, einer erfahrenen und mitfühlenden Lehrerin und Therapeutin für lautsprachliche Erziehung, die Sa- 
rahs Sprachentwicklung stark unterstützte und positiv beeinflusste. Die Therapeutin bereitete die Eltern professionell von Anfang an, ohne es zu verschönern, auf alle möglichen Probleme vor, daher auf höchste Konzentration und harte, langjährige Arbeit bei der Erziehung des Kindes:

„Das Leben wird Ihr Kind in Glaswolle wickeln! Ihr Kind wird immer kämpfen müssen! Darauf müssen Sie es vorbereiten!“ war die Direktive der Therapeutin. Diese Ratschläge haben sich in Sarahs Leben prognostisch bewahrheitet (so ist das Leben).

Die Therapeutin gab den Eltern auch sehr konkrete, praktische Anweisungen zur Vermittlung der Kommunikation und Sprache an. Im Klartext, ohne es zu verschönern, hieß es folgend:

Das gehörlose Kind soll Sprache anschaulich, am besten im Alltag lernen, in der Umgebung der Verkehrsgemeinschaft, also nicht nur Fakten beschreiben, auch Gefühle, Emotionen, Empfindungen, um später Erlebnisse einzuordnen, seelische Regungen, Einstellungen und das Verhältnis zur Umwelt wahrnehmen und artikulieren zu können. Wichtig muss nicht nur die korrekte Aussprache einzelner Worte sein, auch die Intonation des gesamten Textes muss situationsadäquat profiliert (ausgedrückt) werden. Daher muss sehr emotional erzählt werden, damit der Inhalt überspitzt, übertrieben vermittelt wird und von Sarah verstanden werden kann. Wichtig ist auch das Transferieren, Übertragen von einer Situation auf eine andere. Geschichten müssen so erzählt werden, dass man mit offenem Mund, mit größtem Interesse zuhört. Sprache muss man spielerisch näherbringen. Der Sprachreichtum muss immer etwas über dem aktuellen Entwicklungsniveau des Kindes liegen, nur so wird es in der Lage sein, später Abstraktes zu verstehen und auch sehr anspruchsvolle Literatur zu lesen.

Als Priorität gilt: Dem gehörlosen Kind muss man beim Sprechen immer das Gesicht zuwenden, damit es den Mund des Sprechers sehr gut beobachten kann; die Gegenstände, die man zeigt und erklärt, müssen anschaulich präsentiert werden, auch mit anderen Sinnen wahrnehmen lassen (nicht nur mit den Augen): an den Mund halten, zeigen, erklären, anfassen, riechen lassen (außer Gestik, Mimik, auch Geruch, Duft, Anfassen, Schmecken, Bewegungen üben, auf Entfernung achten usw.). (Neef, 2009: 45-47).

\section{Das Lippenlesen}

Das Erlernen des Lippenlesens begann bei Sarah gemeinsam mit der Ausspracheschulung damit, dass ihr die Mutter das Funktionieren der einzelnen Sprechorgane im frühesten Alter bewusst und anschaulich gemacht hat, d.h., erklärte, welche es sind, wie sie funktionieren, was sie mit dem Luftstrom verursachen, wie und wo Laute und Vibrationen, aus Luftwellen - Schallwellen entstehen. Von Anfang an legte ihre Mutter unermüdlich viel Wert auf das Erlernen der Wort-Betonung, ihr 
den Rhythmus (Sprechmelodie) beizubringen, dazu kombinierte sie Musizieren und Sprachtraining (Wort-Betonung und Text-Rhythmus, Intonation), um der "intonationslosen" Sprechweise mancher Gehörlosen (besonders jener, die die Gebärdensprache benutzen, ohne Intonations-Gefühl) entgegenzuwirken. Das sollte der Weg sein: von Ausspracheschulung (mediale Mündlichkeit) zur Diskursfähigkeit (konzeptionelle Mündlichkeit).

\section{Ausspracheschulung}

Aussprache ist die Wahrnehmung und Erzeugung von Lauten und Lautfolgen. Aussprache besteht aus der phonetischen Realisierung der Laute und Lautverbindungen (Artikulation) und der Produktion der suprasegmentalen Strukturen (Prosodie) (Börner, 1999; Hirschfeld, 2010). Aussprache ist also die lautliche Realisierung gesprochener Sprache, die sich auf zwei Ebenen vollzieht: auf der segmentalen Ebene der Artikulation und auf der suprasegmentalen Ebene der Prosodie. Die Aufgabe, Aussprache an gehörlose Kinder zu vermitteln, bedeutet, ihnen zuerst die Fragen bewusst zu machen: Wo und wie entstehen Laute, wo und wie werden Laute gebildet?

Artikulation ist die Lautbildung, eng verbunden mit (Ein- und Aus-)Atmung und Stimmerzeugung (Phonation). Der Luftstrom wird mit dem Mund eingeatmet, gelangt in die Lunge; von dort aus gelangt er als Ausatmungsluft in den Kehlkopf. Im Kehlkopf werden Merkmale stimmhaft/-los entschieden; im Kehlkopf entsteht auch der Knacklaut sowie der Hauch-Laut. Alle übrigen Laute entstehen aus dem Luftstrom, der aus der Lunge über den Kehlkopf in den Rachen-, Mund- und Nasenraum gelangt. Für die Entstehung der einzelnen Laute sind (bewegliche) Lippen und Zunge verantwortlich sowie (unbewegliche) Gaumen, Gaumensegel, Rachen, Stimmlippen und Nasenhöhle.

Prosodie ist die suprasegmentale Ebene der gesprochenen Sprache. Zur Prosodie gehören: Tonhöhe, Akzent, Rhythmus, genauer gesagt Gliederung und Melodisierung; Sprechmelodie, Lautstärke, Dauer, Sprechtempo, Sprechspannung, oder Pausen. Suprasegmentale Gestaltungsmerkmale der gesprochenen Äußerungen (v.a. Akzentuierung und Rhythmisierung) sind oft für den gewünschten (erwarteten) Ausdruck von der situationsadäquaten Sprechintention verantwortlich. Die entstehenden Laute werden nicht nacheinander, nebeneinander realisiert (etwa wie Morse'-Zeichen oder schriftliche Buchstaben), sondern verschmelzen assimilatorisch ineinander, denn die aufeinanderfolgenden Laute beeinflussen sich gegenseitig (Assimilation).

Dazu noch hängt die präzise Wahrnehmung der Laute von Prosodie ab: Akzent, Sprechgeschwindigkeit und Sprechspannung, was den Empfang von Lauten für Gehörlose besonders schwierig macht. Es wird eine verschmolzene Ganzheit wahr- 
genommen, die Gehörlose auf ganz bestimmte Weise sortieren und nachvollziehen müssen: antizipierend blitzschnell überlegen, welche der mehreren Varianten es wohl sein könnten (für Hörende geschieht es ziemlich natürlich, spontan, total problemlos).

Aussprache (als Artikulation und Prosodie) ist bedeutungsunterscheidend, also für das wirkliche Kommunizieren äußerst relevant. Hörende Kleinkinder lernen sie natürlich, nebenbei, völlig problemlos. In jedem Sprachunterricht muss Aussprache so schnell und ganzheitlich wie möglich gelernt werden, im FSU ist Aussprache das übergeordnete Lernziel. Es ist auch das ganz besonders wichtige Lernziel für Gehörlose. Bei der Aussprache-Schulung müssen auch klare Lernziele aufgestellt werden: Welche lautlichen Elemente und Prozesse müssen gelernt werden? Nach welcher zielsprachlichen Norm? Wie nahe soll der Lerner dieser Norm kommen?

Aussprache ist eine grundliegende Teilaktivität des kommunikativen Sprechens, denn Aussprache ist als Vorbereitung auf das Kommunizieren aufzufassen, als Grundlage und Ausgangsbasis, somit als Mittel zum Zweck der guten Verständigung. Aussprache ist ganzheitlich als Artikulation mit Prosodie zu erlernen und zu üben. Gelenkte oder freie Sprechübungen sind immer auch Ausspracheübungen (in denen auf Artikulation zusammen mit Prosodie geachtet werden soll).

Aussprache und Hören sind zwei miteinander eng verbundene kommunikative Teilaktivitäten, um sich im Hörverstehen zu üben. Es wird gesprochen, um zu empfangen, also um hörend zu verstehen. Deshalb ist korrekte Aussprache für den potentiellen Empfang kommunikativ äußerst wichtig, von der korrekten Aussprache

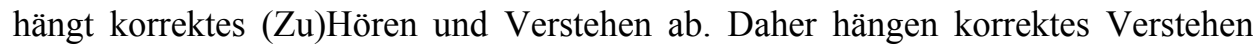
und korrekte Aussprache sehr eng zusammen, denn sie ermöglichen, am Gespräch kommunikativ (intendiert und situationsadäquat) teilzunehmen. Aussprache und Hören determinieren das Lesen und Schreiben. So wie Aussprache Wahrnehmung und Erzeugung von Lauten und Lautfolgen ist, so sind Lesen und Schreiben Wahrnehmung und Erzeugung von Buchstabenfolgen. Also ist das Hörverstehen die Vorbereitung für Lesen und Schreiben. Erst alle vier Teilaktivitäten machen Sprache als gesamtes Kommunikationsmittel aus. Gemeinsam diesen Sprachtätigkeiten ist, dass sie zwar die Zeichenform, nicht aber den Zeicheninhalt der Sprache zum Gegenstand haben, aber sie beeinflussen den Zeicheninhalt gewaltig! Das bedeutet, alle vier Teilaktivitäten sollten ziemlich eng miteinander gelernt werden, denn sie beeinflussen einander und alle vier sind kommunikativ relevant. Daher sind verwandte Lernziele für Aussprache das Hören (für das Hörverstehen), folglich für das Lesen und Schreiben.

Da Gehörlose des Hörverstehens beraubt sind, muss ihr Aussprache-Lernen mit bewusstem Atmen beginnen: Luft einatmen, ausatmen, um zu erkennen, welche Wege der Luftstrom geht, welche Rolle das Zwerchfell beim Atmen spielt, wie sich Vibrationen in verschiedenen Atmungs-Konstellationen anfühlen, welche Lautschwingungen welche Laute entstehen lassen? Es muss begriffen und unermüdlich 
geübt geübt geübt werden, Aussprache als Artikulation und Prosodie auf der Basis der Bedeutungsebene. Das heißt, gelernt werden muss unbedingt an lexikalischen Einheiten sowohl die Lautbildung (phonetische Ebene) als auch die suprasegmentalen Strukturen der Prosodie (Intonation, Wort- und Satzakzent und Rhythmus, die als Kombinationen von Tonhöhe, Lautstärke, Länge, Lautqualität, Pausen).

Auch erklärte ihr die Mutter, dass Sprache nicht nur zur bloßen Verständigung da ist (als Vermittlung von Informationen), sondern auch subtile Ausdrucksmöglichkeiten für Gefühle, Emotionen in Poesie, Ironie, Mehrdeutigkeiten, Witze, Sprachspiele usw. darstellt. Ebenso erklärte sie Sarah lautmalerische Charakteristika, onomatopoetische Ausdrucksweisen (wie dröhnen, kreischen, zittern, rauschen); die Bedeutung von Redewendungen erklärte ihr die Mutter stets im alltäglichen Kontext. Die Mutter half ihr auch beim Erlernen der nonverbalen Kommunikation, auch verschiedene kulturkundliche Begriffe und Abstrakta zu verstehen, dass der Mensch ein Kulturwesen ist (homo cultus), was sich in Sprache widerspiegelt durch bspw. Taktgefühl, verschiedene Lebensweisen, Religionen, Völker, eigene Nationalhymne, Nationalflagge, Mentalität usw. Vieles lernte Sarah zu begreifen, weil sie damit selbst in Berührung kam und daran teilgenommen hat.

Ihre Mutter wusste: Gehörlose können neuen Wortschatz kaum zufällig lernen (was für Hörende das Normalste ist): jedes Bedeutungs-Detail muss von ihnen verstanden werden, also muss es von jd gründlich erklärt werden und dem Kind bewusst gemacht werden. Jedes Wort muss gemeinsam mit der korrekten Aussprache (Artikulation und Prosodie, Wort-Betonung und Text-Intonation) sowie Schreibweise vermittelt werden. Diese Rolle übernahm ihre Mutter, indem sie Sarah jedes lexikalische Detail anschaulich erklärte. Sarahs ständiges Herantasten an die Sprachwelt der Hörenden und ihr Ausprobieren mit neu erlernten Wörtern, das war ihr mühevoller und zeitaufwendiger Alltag. Denn sie wusste, jedes Sinn-Defizit, jedes NichtVerstehen ist ein unwiderruflich bleibendes Kommunikations-Problem; deswegen erkannte sie, alles was sie lernt, hilft ihr, in der Zukunft selbständig zu sein und selbständig zu leben. Sarah bestätigt, es ist unsinnig zu meinen, ein Kind ist für dieses oder jenes Wort zu klein.

Die Mühen ihrer Eltern erweiterten Sarahs Horizont, ihr Weltverständnis. Alle Spielabenteuer prägten ihre Vorstellungskraft und Phantasie, umso mehr, als Sarah schon als Kind sehr viel Entdeckungslust und Bewegungsbedürfnis sowie Neugier und Interesse an der Welt zeigte. Glücklicherweise hatte sie auch sehr viel Spaß, Freude, Ausdauer und Konsequenz am täglichen Üben.

\section{Sarah`s Kompensationsmöglichkeiten}

Bei Sarah haben sich sehr bald zwei Sinnesmodalitäten als Ersatz-Reize entwickelt: 
- die Empfindlichkeit für Laut-Schwingungen, Vibrationen (erbrachte, ergab das Horchen, was rhythmische Tanz-Bewegungen ermöglichte),

- breitperspektivisches Sehen mit Augen (für das Ablesen vor allem der Mundbilder, aber auch von Gestik, Mimik und der gesamten Körpersprache anderer).

Sie musste sensorisch kompensieren, um zu kommunizieren. Beide helfen ihr bis heute, nicht nur lautlich zu sprechen, sondern auch Musik wahrzunehmen und Tanz musikalisch auszuleben. Sarah lernte mit allen Sinnen. Das unzureichende Funktionieren des Hörvermögens wird durch das verstärkte Funktionieren eines anderen (korrekt funktionierenden) Sinnes ausgeglichen, kompensiert, d.h., die sensorische Dysfunktion muss kompensierend ersetzt werden, mit Einsatz anderer Sinne, die korrekt funktionieren. Das lautliche Sprechen-Lernen war für Sarah mit ihrem ganzen Körper bewusst: so gelang sie langsam aber sicher zum Erreichen der korrekten Aussprache (Artikulation zusammen mit Prosodie, also zur medialen Mündlichkeit). Die Sprachproduktionen anderer nachzuvollziehen, also Sprache zu empfangen, bedeutete für sie, mit den Augen hauptsächlich durch Lippenlesen, aber auch durch Beobachtung der Körpersprache anderer Kommunikationsteilnehmer zu realisieren. Dazu kam ihre Empfindlichkeit (Sensibilität) für Schwingungen, mit ihrem ganzen Körper Vibrationen zu empfangen und zu deuten zu lernen. So bekam sie Ahnung vom Rhythmus der gesamten Geräuschkulisse (das nennt sie Horchen).

Von Anfang an, als sie mit zwei Jahren die Sprachschule besuchte war sie von Musik und Tanz angeregt und begeistert, fing Sarah an, Instrumente zu spielen: Xylophon, Triangel, Flöte, Harfe, Trommel, später auch Klavier. Sarah spielte Instrumente, um richtig zu atmen; sie entlockte ihnen Geräusche, die sie zwar nicht hörte, aber deren Schwingungen sie spürend wahrnahm. Flöte zu spielen half Sarah, ihren Atem zu kontrollieren, um die korrekte (akzeptable) Aussprache zu lernen (Artikulation und Prosodie). Zur Atemkontrolle gehörte auch das richtige Atmen durchs Zwerchfell sowie Betonungen zu bilden. Die richtige Betonung ist oft wichtiger für das Verstehen des Gesprochenen als die korrekte Artikulation und Prosodie, ebenso für korrektes Schreiben. Das wurde gleichzeitig die Basis für das Erlernen des Lesens, der Rechtschreibgewohnheiten und des kommunikativen Schreibens ihres Idiolekts.

Es ist nur verständlich, dass Gehörlose Lernprobleme nicht nur mit lautlicher Sprach-Produktion haben, sondern auch (folglich) Schwierigkeiten mit der Schriftsprache in der Muttersprache, daher ist die Schriftsprache für gehörlose Kinder ziemlich kompliziert, vergleichbar mit Hörenden, die eine Fremdsprache lernen. Geschriebene Texte Gehörloser ähneln denen der Ausländer, die Fremdsprache lernen: unvollständige Endungen, unkorrekte Fälle, Rektion, Syntax, lexikalisches Unverständnis, Rechtschreibung, aber auch semantisch ungelungene Ausdrucksweisen, die aus Unkenntnis des kulturellen Hintergrunds stammen, sind die meisten Fehler. Wobei: Ausländer sind privilegiert, sie erfahren Sympathie und Entgegen- 
kommen seitens der Muttersprachler, auch deswegen, weil ihr Sprachstand sich eventuell bessern kann (Ehlich, 1982; gr. xenos: Fremder=Freund).

Aussprache als Lautproduktion bedeutet, mediale Mündlichkeit lernen. Sarah bezeugt, für jemanden, der nicht hören kann, bedeutet es stellvertretend lernen, bewusst zu fühlen, wo und wie Sprachlaute eingesetzt werden, wo und wie sie entstehen, wie verhalten sich die einzelnen Organe zueinander. Um zu verstehen, wie Laute entstehen, musste sie zuerst begreifen, wie und welche Organe einzeln und/oder zusammen kompakt funktionieren, um Laute, die sie nicht hört, produzieren zu können. Sie musste zuerst erlernen, sich vorzustellen, wie sich die einzelnen Laute anhören (sollen). Erst dann versuchte sie, mit Hilfe von Vibrations-Technik, Laute (die sie nicht hört, nur vibrierend fühlt) selbst zu produzieren (auch Karpińska-Szaj, 2013; 2015). Helen Keller, eine taubblinde Schriftstellerin, bezeugte, jedes Atom ihres Körpers sei ein Vibroskop (Neef, 2009: 150).

Wer nicht hören kann, muss lernen, bewusst zu fühlen, um nachzuempfinden, wie Sprechlaute eingesetzt werden, um zu erahnen, um sich antizipierend vorzustellen, wie sich die Laute (kombiniert in Worten) wohl anhören; das erfordert bewusstes Wissen über den Einsatz der einzelnen Sprechorgane am Sprechakt, bspw. die Zungenstellung, Gaumen, Lippen. Ebenso muss über die Rolle der Atmungswege wissen, weil korrekte Atmung mit Einsatz des Zwerchfells rhythmisches (Aus)Sprechen erleichtert (bzw. überhaupt ermöglicht).

Die Unterschiede zwischen Hörenden und Nicht-Hörenden lassen sich bildlich an ihren Ausdrucksmöglichkeiten ihrer Wahrnehmung im Schlaf veranschaulichen: Hörende sprechen träumend im Schlaf, Gehörlose drücken ihre Träume mit Gesten und Bewegungen der Gebärdensprache aus.

\section{Aussprache dreispurig gelernt: lautliches Sprechen begleitet von Musik und Tanz}

Mit drei Jahren lernte sie lesen und erlebte zum ersten Mal mit sehr viel Freude einen Opernbesuch (im echten Opernhaus), beides wurde zum Schlüsselerlebnis! Mit dem Lesen entwickelte sich in ihr das unstillbare Bedürfnis, sich gewissermaßen selbständig zu machen: die Welt zu erfahren, Wissen zu ergründen. Sie genoss unstillbaren Lesehunger. Bücher wurden für Sarah Sprache, Verständnis der Welt, Teilhaben an der Welt. Aber auch eine Wissens-Basis. Im Lesen und Tanzen ging Sarah kommunikativ voll auf. Das dritte Lebensjahr wurde für Sarah die kognitive Wende, die Zäsur, die ihr Leben geprägt hat.

Natürlich ist, dass der Mensch mit allen Sinnen ausgerüstet (ausgestattet) ist und deshalb als Kleinkind ganz spontan und immersiv zu kommunizieren lernt. Schon bei Babys bemerken wir die dem Menschen angeborene Faszination für das Gesicht der Bezugsperson (als Kontaktweise, -fähigkeit, -bedürfnis). Sarah lernte ganzheit- 
lich und anschaulich; das ist der natürliche Weg, wie (Klein)Kinder lernen. Es ergibt sich der Zirkelschluss: Das Wahrgenommene prägt unsere Lebenserfahrung und unsere Lebenserfahrung determiniert weiterhin (rückbezüglich) unsere Wahrnehmung. Das bezieht sich sowohl auf Manualisten als auch auf Oralisten.

Kompensation bedeutet, stellvertretend mit anderen Sinnen wahrzunehmen. Sensorische Dysfunktionen verursachen eine sensorische Ausbreitung, Erweiterung, Übertragung auf den Empfang mit anderen Sinnen. Wer nicht hören kann, entwickelt (unter anderem, wie bei Sarah) die Sensibilität für Vibrationen, also das Horchen, eine sehr behilfliche Kommunikationsfähigkeit, die Geräuschkulisse wahrzunehmen. Wenn Gehörlose Laute seiner Umgebung nicht nachvollziehen, folglich nicht nachahmen und nicht produzieren können (die mediale Mündlichkeit entwickeln), bedeutet es, sie können nicht verstehen. Deswegen können sie auch nicht bewusst kontrollieren, ob korrekt nachgeahmt oder nicht, ob eigene Lautproduktionen der lautlich sprechenden Umgebung angepasst ist. Wie soll man dann an Gesprächen teilnehmen, sprechen, diskutieren, lautsprachlich kommunizieren, überhaupt an zwischenmenschlichen Relationen teilnehmen, wenn man Laute nicht aussprechen kann? Die konzeptionelle Mündlichkeit kann man erst dann aktivieren, wenn man die mediale Mündlichkeit realisieren kann.

„Der gehörlose Mensch horcht mit seiner Seele, die Schönheit der Musik liegt als sinnliche Wahrnehmungen vor. Gehörlose, die sich mit Musik beschäftigen, arbeiten daran, ihre Fähigkeit im Empfangen, im Spüren von Vibrationen zu erkennen und zu verfeinern. Die fühlbaren Schwingungen beschränken sich nicht nur auf den Tastbereich der Haut. Sarah fühlt (empfängt) diese Schwingungen detailliert im ganzen Körper: in der Kehle, im Rachen, an den Zähnen, auf den Lippen, auf dem Gesicht, die tieferen Töne hört sie hauptsächlich in ihren Beinen und Füßen, die höheren Töne an einzelnen Stellen des Gesichts, des Nackens und der Brust. Auch über die Knochenleitung, über die Schädelknochen. Auch auf der Haut und auf den darunterliegenden Gewebeschichten bilden sich empfindungsfähige Zonen“. (Neef, 2009: 54).

\section{Lippenlesen}

Sie kompensiert ihr fehlendes Gehör mit visuellem Hören, d.h., dazu muss den Mund des Gesprächspartners sehen, von vorne oder mindestens von der Seite. Breitperspektivisches Sehen erfordert eine enorme Konzentrations- und Beobachtungsfähigkeit seitens des Gehörlosen! Anstatt zu hören, sieht sie breitperspektivisch. Sie benutzt die Augen, wenn Hörende die Ohren benutzen; das bedeutet, sie muss das Gesicht ihres Gesprächspartners sehen, sobald jemand aus ihrer Umgebung verschwindet, ist die Kommunikation für sie unterbrochen (so wie in der Baby-Zeit, als ihre Mutter ins andere Zimmer ging). Gehörlose sind aufmerksame und antizipie- 
rende Beobachter mit sehr viel Vorstellungskraft (Phantasie) und Intuition ausgerüstet, d.h., wenn sie sehen, dass etwas in Bewegung ist und vibriert, bspw. ein Blatt fällt, erkennen sie es; mit ihren Augen (Seh-Sinn) nehmen sie das wahr, was sie nicht hören können. Die Augen-Wahrnehmung wird ins Gehirn geleitet, wo die notwendige Vorstellung von dem entsprechenden (potentiell begleitenden) Ton dazu erzeugt wird. Sarah hört bspw. das Pfeifen des Windes nicht, auch kann sie es nicht sehen, aber sie merkt (mit Hilfe des Tast-Sinns), wenn ihr die Luft eiskalt mit starkem Druck unangenehm ins Gesicht fährt; dann stellt sie sich vor, dass und wie der Luftdruck ein Geräusch verursacht: so wird die Wahrnehmung durch den Tast-Sinn aktiviert. Dazu muss sie ihre Vorstellungskraft und Empfindungen aktivieren. Lautsprachlich kommunizieren zu können als Gehörlose impliziert, Hörverstehen ohne den Einsatz des Hörvermögens das Gesprochene anderer durch Horchen, also Vibrationen-Empfang der Schallwellen nachzuvollziehen (begleitet von musikalischrhythmischen Tanzbewegungen). Als Sender musste Sarah Aussprache bewusst erlernen, das bedeutet, Artikulation gemeinsam mit Prosodie nachzuempfinden und zu produzieren. Parallel dazu erlernte sie das Lippenlesen, als breitperspektivisches Sehen mit Augen. Das machte sie multimodal kommunikativ (Augen, Bewegungen, Mund), auf den Kommunikationsaustausch mit anderen bereit.

\section{Zusammenfassung}

Im Rahmen der Sprachenpolitik hat jeder das Recht, Sprache(n) zu lernen, um kommunizieren zu können: verstehen, verstanden und akzeptiert werden. Es steht jedem zu, die Muttersprache zu erlernen und darüber hinaus auch Fremdsprache(n) (Grucza, 1999). Sarah Neef machte es möglich, trotz Gehörlosigkeit, ihre Muttersprache lautsprachlich zu lernen, und darüber hinaus einige Fremdsprachen. Zu postulieren ist, innerhalb der Sonder-Glottodidaktik Bedingungen für inkorporierendes Lernen (pol. nauczanie właczające) (nach Karpińska-Szaj (2013), Domagała-Zyśk und Karpińska-Szaj (2011)) zu erarbeiten, um Lernern mit sensorischen Dysfunktionen den Fremdsprachenunterricht leichter zu machen. vgl. zu ermöglichen.

\section{Bibliografie}

Bonacchi, S. 2011. Höflichkeitsausdrücke und anthropozentrische Linguistik. Warszawa: Euro-Edukacja.

Domagała-Zyśk, E. und K. Karpińska-Szaj. 2011. Uczeń z wadq stuchu w szkole ogólnodostępnej. Podstawy metodyki nauczania języków obcych. Kraków: Impuls.

Grucza, F. 1993. „Zagadnienia ontologii lingwistycznej: o językach ludzkich i ich (rzeczywistym) istnieniu". In: Bartmiński, J. (red.) Opuscula Logopedica. In honorem Leonis Kaczmarek. Lublin: UMCS. 25-47. 
Grucza, F. 1999. „Współczesna polska polityka językowa w zakresie nauki języków obcych”. In: Mazur, J.(red.). Polska polityka językowa na przełomie tysiacleci. Lublin.73-98.

Grucza, F. 2012. „Zum Gegenstand und zu den Aufgaben der Anthropozentrischen Linguistik, Kulturologie und Kommunikologie sowie zur gegenseitigen Verentzung dieser Ereknntnisbereiche“. Kwartalnik Neofilologiczny LIX 3/2012. 227-344.

Karpińska-Szaj, K. 2013. Nauczanie języków obcych uczniów z niepetnosprawnościq w szkołach ogólnodostępnych. Poznań: UAM.

Olpińska, M. 2009. Nauczanie dwujęzyczne w świetle badań i koncepcji glottodydaktycznych. Warszawa: Euro-Edukacja.

Rutkowski, P. 2016. „Migając poznają świat”. Przewodnik katolicki 2016/34. 35-37. 